Chapter 8

\title{
Emotion, Affection and \\ Maternal Speech in Parental Care
}

\author{
Deise Maria Leal Fernandes Mendes and \\ Luciana Fontes Pessôa \\ Additional information is available at the end of the chapter
}

http://dx.doi.org/10.5772/57337

\section{Introduction}

This chapter discusses the role of emotions and affective communication in practices of care and the relevance of these components in children development. The importance of considering the different development trajectories as a result of different parental practices that arises from the culture is also discussed. Affection and emotion are considered crucial dimensions of parental care with consequences in child development through daily practices, parental beliefs, and expectations that guide the way to raise and to educate children. In the first year of life, children's participation in mother-infant's interactions, as the free-play situations, involving emotional expressions and vocalizations, is considered a prominent factor for the development of affection, attention, communication and cognitive functioning. Evidence accumulated in recent decades has highlighted the role of affective exchanges between parent and their children for a healthy development. Also, mothers' affective speech and the process by which the language development is constructed or acquired in this interactional context has been subject of various theoretical formulations and gave rise to a diverse literature of empirical investigation. The formulation of models and assumptions about human development requires an understanding of these processes and the factors associated with them. So, we discuss here these mechanisms in the early stages of ontogeny. Arguments are based on results of some international research and current evidence of Brazilian studies.

\section{Development trajectories in different eco-cultural contexts}

Evolutionary and cultural approaches in psychology, taken from a complementary and integrated way, provide us a powerful theoretical synthesis for a broader understanding of 
human development. According to this view, it's reasonable to consider that babies are born biologically prepared to participate and be agents of a social matrix from an early set of skills that allow them to be sensitive and interact with their caregivers. At the same time, from birth they are immersed in culture and become cultural agents. As discussed by Seidl-de-Moura and Mendes (2012), the possibilities and limitations of these competences seem to prepare the infant to interact with parents that have practices of care derived from behavioral predispositions and beliefs.

Parental beliefs and practices of care are understood as been forged by culture, and are associated with goals of development. In all cultures, as evidence from cross-cultural studies (Keller, 2007), parents have the goal that their children develop in a healthy way, and become successful adults. This idea of personal fulfillment is linked with individual, family and cultural values and rules. It is in the developmental niche, a concept of development context formulated by Harkness and Supper (1994), that socialization trajectories are built, grow and change over time.

A component of this proposed system is the psychology of the caretakers, and part of this are the parental ethnotheories (parents' cultural belief systems), that are the nexus through which elements of the larger culture are filtered. They are an important source of parenting practices and the organization of daily life for children and families. As an organized set of ideas that are shared by members of a cultural group, they are often implicit, and have strong motivational properties for parents. Harkness et al. (2010) argues that understanding parents' ethnotheories about their children is essential to understand the strategies parents use to help their children grow up and be successful.

Keller (2007) in her work also emphasizes the role of parental beliefs and goals in parental practices and in the child development. According to her, the baby presents great cerebral plasticity in the first two years of life and their interactions with caregivers in this period are organized according to the predominance of different parental systems. These systems are universal and result of propensities to care. However, they are manifested in different ways according to cultural diversity.

The Component Model of Parenting defined by Keller (2007) is conceptually composed by six universal and independent systems: primary care, that involves a set of activities that aim at meeting babies' survival needs, including health related activities, such as nursing, washing, and so forth, with the function of to reduce stress and promote security and trust in relation to caregivers' protection; body contact system, which promotes corporal contact, and involves carrying the baby close to the body, affecting the bonding between mother and baby and group cohesion, and protecting the baby from dangers; body stimulation system that involves any motor, kinesthetic, tactile and balance stimulation of the baby, promoting communication through the body; object stimulation presents the object world and physical environment to the child, and is related to exploratory activities; face-to-face context is characterized by mutuality through eye-gazing; and narrative envelop, which refers to the symbolic mediation that involves the infant through mothers' conversations. 
Evidence produced by studies developed by Keller and collaborators (Keller, Borke, Yovsi, Lohaus, \& Jensen, 2005; Keller, Borke, Lamm, Lohaus, \& Yovsi, 2011) indicate that culturespecific parenting strategies differ with respect to the type and amount of behaviors expressed, and also to the developmental course of particular behaviors. Different channels are chosen to express socialization strategies, and therefore, caregivers emphasize these systems of parenting differently, depending on the cultural contexts. Variation is manifested in relation to their care practices, and to their beliefs and parental ethnotheories.

These systems prompt different socialization experiences with different psychological consequences to the children. Parent behaviors within these different systems may vary in terms of contingence and warmth. Keller (2007) argues that parenting is the main reason why individuals in different cultures differ from each other. Consequently, it has been considered as a significant feature of culture, constituting the largest mechanism for the transmission of values and practices through the generations.

Cultural differences in terms of parental ethnotheories and interactional mechanisms, especially between family members, are substantial. However, in general, mothers are the main significant figures to take care of babies in the first years of life (Keller, 2007). In this way, parents, specially the mothers, define socialization trajectories and the process of self development. These pathways are designed from individual characteristics based on culture. The socialization of emotion in the first year of life and the acquisition of language will be assessed according to this approach.

\section{Emotional development and social interactions in early stages}

Diverse eco-cultural contexts with its specificity of possibilities and challenges, as discussed, provide differences in terms of parental systems and developmental trajectories for children. As the same time, human development is conceived as a process in which human beings are born as cultural beings that look for interpersonal interaction. In the familiar niche they start social interactions with their parents. This way, family constitutes the centre of their lives in the early stages and can be considered as mediating the relation between child and environment. This notion is contemplated in some important conceptions of developmental context as that of Harkness and Super (1994) and Bronfenbrenner (1996), in which the family is part of development microcontext. This is the context in which children begin to deal with their emotions and those of others.

The importance of interpersonal relations in the lives of children, in particular the emotional and affective exchanges with their mothers, has been highlighted since Bowlby (1969) and the attachment theory. Besides his work, Harlow (1958), studying chimpanzee infants, demonstrated that contact comfort (with a wire mother that was covered with cloth over a wire) was more important for the infant than the hunger drive. Even well before, in an evolutionary perspective, Darwin devoted a book to the emotions and their adaptive role in the life of man and animals. 
In more recent publications, authors as Reddy and Trevarthen (2004) suggest that emotions are the key to psychological engagement, since they are part of us as individuals in our active and assertive relationships and it's part of their nature to be intensely shared. As they said "For all who deal with infants, emotional engagements with them provide the most informative as well as the most helpful route to understanding them." (Reddy \& Trevarthen, 2004, p. 3)

Capacities required for the perception and expression of emotions have been considered fundamental for the quality of early interactions, which in turn are a rich context to promote affective and social development. This early emotional involvement indicates an increase in "awareness" by babies, in relation to their ability of attention and intentions, and to other people's expectations.

Authors from different areas as psychologists, psychoanalysts, and neonatologists raised important questions in relation to the potential for emotional experiences in early life of babies in the womb and their impact in the fetus development (Cunha, 2002; Piontelli, 1992). According to them, positive and negative emotional experiences intra utero make a difference in an infant's development. Our brain develops in direct response to our mother's experience of the world. This way, if a fetus is continually exposed to mother's stress (with the release of cortisol in her bloodstream) it's observed an accelerated fetal heartbeat and hyperactivity. It is still considered certain continuity between intrauterine and earliest infancy, since some children's behaviors seems to maintain associative links with favorite moves and postures showed in the womb.

Recent advances of Neurosciences indicate that since 20 to 24 weeks of prenatal life, when the neuronal migration is complete, the infant can perceive the surrounding environment and construct proto-representations arising from experiences and emotions that will "sculpt" his/ her neural networks. Therefore, it can be thought that it is possible the registration of positive affects sensations that will generate feelings of security and welcomed, and if continuous and strong enough can "sculpt" long-lasting neural circuits. The counterpoint of this can be thought of in relation to negative affects sensations.

After birth, infants and their parents regulate their interactions through the baby's emotional expressions and the parent's behaviors and reactions. The context of affective interchanges in the early stages is considered crucial to the main primary achievements that a baby must make. Since the first moments of life, human being show different emotional expressions, and from a very early age they seem oriented to other person, as a social partner.

Even newborns, when observed, show a variety of emotional behavior. If they are upset, when they feel alone, or in need of food and attention, they cry and express discomfort. They look carefully for people and objects around them, and when they are looked at by someone with joy and treated with care they seem to manifest wellness and positive emotions. At this moment, they show relaxed and often smile. In fact, they smile from birth or before, and with a few weeks of life they begin to smile the "social smiles" (Mendes, Seidl-de-Moura, \& Siqueira, 2009; Messinger et al, 2002). Before long, they begin to react contingently with smiles to affective behaviors of their mothers (Mendes \& Seidl-deMoura, submitted; Mendes et al., 2009). Maternal smiles proved to be powerful elicitors of smiles on baby, but also other affective behaviors. Among them, mother speech/vocaliza- 
tion directed to the baby drew attention for being the only one that elicited responses in contingent smiling babies up to two months of life.

Although displaying a variety of facial expressions and body postures to express emotions, the set of distinct emotions the newborns show can still be considered as limited forward to the diversity of human emotions. The changes observed over the early years, however, are such that after a few months of born, babies exhibit a wide range of emotions. In fact, at around three years of age, one can consider that is present in a child almost any variety of adult emotions (Lewis, 2010). For three years, approximately, the changes observed in this set of human emotions and in their view forms are such that more than a few emotions and their manifestations for many and highly differentiated, with a vast repertoire of demonstrations, of more direct to that denote certain subtleties.

Based on an evolutionary and socio-cultural approach to understand human development, all this behavioral and emotional expressions repertoire which diversifies in a few years, seeks to account for developmental needs and requirements. In addition, to meet the requirements and challenges of a cultural context in which the child develop is adaptive in that it seems to have evolved according to our phylogeny, to promote the survival and future reproductive success of individuals. In this sense, we can consider the sensitivity to social stimuli and social relations with co-regulation of affection, feelings and emotions as the basis of socio-emotional development. Parent's beliefs and goals about emotional experiences and expressions shape the process of socialization of emotion.

\section{The socialization of emotion: parental goals and expectations of children caregivers}

The interactional characteristic that human development has, as already commented, is especially evident in the emotional development. Emotions are powerful resources that humans have to deal with situations experienced and with interpersonal communication. They can also be understand as a mental system of self-regulation, responsible for the regulation of the concern-relevant aspects of an individual's actions, what means that they have a complex function in human action regulation (Holodynsky \& Friedlmeier, 2010).

Emotions are fundamental to children learn to understand themselves and the other in the social world they live. Since before the language acquisition, emotional communications are fundamental in the social interactions. The socio-cultural context constitutes the meaning and the ways to express emotions. In all the cultural groups, parents want to pass on strategies that promote the survival of their children and their cultural competence. As primary socialization agents, parents can guide their children in how to express their emotions and how to "read" the other's emotions, while culture provides guidelines of how to raise children and general patterns of emotion decodification and expression.

Theoretical models that focus on understanding of the emotional socialization process emphasize how ideas about emotions and values of parents influence their choices of practices 
and strategies of emotion socialization (Dix, 1991; Eisenberg, Spinrad, \& Cumberland, 1998). It's also necessary to keep in mind the fact that this socialization process is a two-way process, and, as well as practices of care have effects on the emotional and social competence of children, their behavior and reactions are likely to have repercussions in how their caregivers act and give them orientations. To examine parents' goals and beliefs about emotions an interesting alternative can be to consider the familiar context that plays an important role in the emotional development. Results from Brazilian studies and investigations carried out in other countries underlines how much the actions of parents are important to the processes of comprehension and regulation of emotion of their children.

As argued by Eisenberg et al. (1998) the existing set of available evidence provides support for the view that parental socialization practices have effects on children's emotional and social competence. Particularly, parents play an important role in guiding children's earliest efforts to regulate negative emotions. This way, it's possible to think that socialization of emotion regulation includes mothers' responses to children's distress as well mothers' modeling and use of emotion regulation behaviors.

Mirabile, Scaramella, Sohr-Preston, and Robison (2009) were interested in examining the extent to which children's (2-year-old) propensity towards negative emotional reactivity moderated the relationship between mothers' socialization of emotion regulation and children's use of specific emotion regulation. It was found that mothers with less reactive children who used more soothing had children who were more likely to use interactive, distraction-based regulatory behaviors during a frustration situation. It was also observed that children's propensity towards negative reactivity significantly interacted with mothers' use of physical soothing. It was argued that the effects of mothers' socialization behaviors and children emotion regulation emerged, suggesting that mothers parenting behaviors, specifically their efforts to socialize emotion regulation, influenced children's ability to regulate frustration.

In other study, McElwain, Halberstadt and Volling (2007) show that mothers' and fathers' supportive reactions together contributed to greater coordination between child and a friend (3- to 5-year-olds) when they are playing, during a sharing task. Further, for boys only, when one parent reported low support, greater support by the other parent was related to better understanding of emotions and less intense conflict with friends.

Lunkenheimer, Shields and Cortina (2007) observed the positive emotion socialization practice of parental emotion coaching and the negative socialization practice of emotion dismissing during a family interaction task and examined their effects on children's emotion regulation and behavior problems in middle childhood (children aged 8-11 years). Outcome measures included mother and father reports of emotion regulation and behavior problems, and it was verified that emotion dismissing was a risk factor, contributing to poorer emotion regulation and more behavioral problems.

Previous research indicates that the quality of parenting during early childhood impacts children's ability of self-regulation of emotion within the context of childcare settings (Nicholson \& Artz, 2006). The trend of parents act as advisors and transmit knowledge about emotions increases the ability of the children in relationships with peers, and their capacity to 
understand and talk about emotions (Denham \& Kochanoff, 2002). Besides that, proactive strategies of parents as encouraging presence, clear instructions and placing limits, contribute to the reduction of problems related to externalizing emotions in preschool children (Denham et al., 2000).

Cross-cultural studies are crucial to examine this process in various cultural systems. Even among cultures within the same country in which one might expect that there was much in common evidence show striking cultural differences determining development goals that differ so markedly. It's the case of Tamang and Brahman Nepali children that have culturally specific emotion scripts that may reflect different emotion socialization experiences.

To study emotion socialization and children's ideas about emotional displays the child-adult interactions of children (3-5 years old) were observed and village elders, as well as, first-grade boys and girls (aged 6-9 years) were interviewed in Tamang and Brahman villages (see Cole \& Tamang, 1998; Cole, Tamang \& Shrestha, 2006). Tamang practices are consistent with their view that competent children are socially graceful and never angry. Tamang rebuke the angry child but reason with and yield to the child who appears ashamed. For the other hand, Brahman practices appear to be consistent with the privileges and duties of high caste status, and the principles of Hinduism. Brahmans respond to child anger with reasoning and yielding but ignore shame.

Tamang and Brahman caregivers socialize children's anger and shame differently because of their cultural values. Brahman children, whose mothers reported teaching their children about emotion, were more likely than Tamang children to endorse negative emotions and to report masking negative emotion. Differently, Tamang mothers reported that children learned about emotion by themselves. These differences appeared to be related to socialization processes in the respective cultures.

Studies with mothers of preschoolers in China and India reported on the value they accorded to items tapping two socialization goals, Filial Piety and Socioemotional Development and two parenting styles, Authoritative and Authoritarian. According to Rao, McHaleb and Pearson (2003), there were found positive associations among these three variables for Indian mothers, reflecting Hindu beliefs about young children and childhood. Among Chinese mothers, on the other hand, Filial Piety was negatively related with Socioemotional Development and the use of Authoritative practices. Authors argued that Chinese mothers believe that the use of authoritative practices, which encourage socioemotional development in children, will inhibit achievement of filial behavior and academic achievement.

Brazilian studies with the focus on emotion socialization are rare. One of them (Mendes \& Cavalcante, submitted) reported some beliefs and expectations of 60 dyads composed of a mother and other child care providers (20 dyads mother-grandma, 20 dyads mother-nanny and 20 dyads mother-childcare workers) about emotion expression in infants. They were caregivers of infants up to one year of age, $56.7 \%$ of which was girls.

According to the results, all the participants considered the smile an important facial expression for infants and think that smiling is important to the child express their emotions and feelings. They, in general, reported higher values for the age at which each emotion emerges 
to which the literary indicates. It was also found that mothers were more attentive and concerned than other caregivers, with the necessity of a capacity of emotional regulation by babies. Mothers thought they need to develop this capacity during the first three years of life, especially, those who have their children, part of the day, in a nursery.

The concern of mothers and caregivers in general with the different ways of emotional development is related to the influence of the psychology of the caretakers and health practices they adopted in the early years of life of the children. However, as discussed, ethnotheories and practices of care reflect cultural models prevailing in societies where the child and their different caregivers live, and have implications for emotional competence to the development.

\section{Language: What does it mean for the human development?}

The contact with members of the linguistic community since the first days of a child's life is of basic importance to language development. An important part of information that developing babies process is the linguistic input of members of their community. As Keller (2007) points out, one of the components in the parental system of care is the narrative envelope. Babies are wrapped in this narrative envelope and their development processes are immersed on it, including language acquisition. Focusing on one aspect of the narrative envelope, maternal speech directed to children has been studied for decades in order to understand the process of language acquisition.

From a socio-cultural perspective, one factor of great importance in the development of children's linguistic capacity is the language of members of their socio-cultural context. Vila (1995) emphasizes that the experience of language usage in interpersonal interactions, especially with adults, is the child's main source of information about language itself, about his/her culture, and about the role of language in the culture in which he/she is inserted. From the linguistic input it is possible to identify the speaker's community, to transmit socio-cultural models, and to adapt the use of the language to the community the individual belongs.

The characteristics observed in the structure and the type of maternal speech directed to the child indicate that it constitutes a type of differentiated input (motherese), which intuitively considers the child's age, his/her language development stage, and mother's conceptions and expectations regarding the child's level of development.

In the beginning of last decade, some studies, as Chapman (2000) focus on the individual process of infant language acquisition, considering historical events and variations of the environment evidencing that the research of genetics and input of the environment can both contribute to an interactional perspective. The author has stressed out the importance of the relationship between natural and cultural aspects in the functioning of the linguistic system, and concluded that initial language development must be seen as the integration of multiple aspects, some of them learned at the beginning of the development.

Also, Hoff and Naigles (2002) had investigated vocabulary production in 63 American dyads in linguistic interactions in two different moments (when the child turned two years-old and 
ten weeks later) during twenty five minutes observation sessions. Results indicated that the production of maternal speech in quantitative terms, with a rich lexicon and with complex semantics, can bring significant benefits for the lexical development of children.

\section{Acquisition of language in the first two years: the role of the mother speech}

Discussing the acquisition of language, several studies have focused on the role of maternal input/speech in the process of language acquisition (Weizman \& Snow, 2001; Camaioni \& Longobardi, 2001; Lidz, Gleitman \& Gleitman, 2003; Pessôa \& Seidl-de-Moura, 2008; Pessôa \& Seidl-de-Moura, 2011).

Camaioni and Longobardi (2001) examined speech characteristics of fifteen Italian mothers of high middle-class, in a longitudinal study when the children had four and eight months-old, in three specific contexts: playing with familiar toys, playing with new toys, and being fed. It was observed that mothers produced more verbs than substantive in all the analyzed contexts. According to the authors, this result may have a direct relationship to the morphology of Italian language and the structure of the sentences in this language. Results indicate the importance of the knowledge about the syntactic structures of the studied language. This structure will influence the type of sentences emitted by the mothers to their children and their process of initial language acquisition.

In a cross-sectional study carried out by Bornstein and cols. (2004), which aimed at identifying characteristics of the vocabulary of 20 months-old children in Spain, Germany, France, Italy, Korea and United States, the prevalence of substantives, followed by verbs and adjectives was observed. One can think of how cognitive, linguistic and experimental factors, or the association of all these aspects, can contribute to these results. The cultural aspect cannot be left untouched when analyzing the results found in this study. From the moment we hypothesize the influence of maternal input in both the process of language acquisition and the phenomenon of motherese - in which the mother adjusts her speech to the period of child's development - it can be assumed that, in general, mothers use more substantives in her speech and does not have yet the concern with the correct structures of the phrases, as well as the role played by verbs.

It seems that some of the differences observed in maternal characteristics are perhaps a result of cultural and individual aspects, strategies and maternal expectations. On the other hand, children can develop different forms of appropriating the information presented in Child Directed Speech (CDS), and this process may be related to their own individual characteristics.

The literature indicates that different aspects in maternal speech have been investigated in cross-sectional studies since the 1970s. For instance, pragmatic characteristics of maternal speech had been identified in different social classes and contexts and in different children's age levels. Syntactic and semantic aspects of maternal speech and their relationship to the development of language have also been considered. The effect of the frequency and structure 
of maternal input in the development of child language, the effect produced by different types of maternal type input in distinct classrooms and in different cultures had also been studied. The primary role played by linguistic interaction in the process of language acquisition and the importance of games and social exchanges in this process had been identified, as well as the importance of joint attention in the development of initial language.

Between 10 and 24 months of age, children progress from communicating through conventional signals to communicating through symbols in a variety of situations. Few longitudinal studies focusing on this transition, considering mother-child dyads longitudinally, and using frequent observations over a lengthy period of time have been conducted (i.e. Camaioni, Aureli, Bellagamba \& Fogel, 2003; Pessôa \& Seidl-de-Moura, 2011).

One important longitudinal study had been carried out by Bornstein, Tal and Rahn (1992). In this study the authors analyzed and compared the language used by mothers with babies of 5 and 13 months of age in four different cultures. The two main aspects of language consisted in informative data, which involved direct questions, phrases, etc., and affective aspects, which corresponded to the use of onomatopoeic sounds, not propositional, rhyme, sounds of animals, etc. Data had evidenced, practically for all cultures, a larger use of speech reflecting affective aspects to the five months-old babies, and the use of more informative language to the thirteen months-old babies. This indicates mothers' sensitivity to the developmental characteristics of their special interlocutors.

Considering that languages and cultural characteristics may influence these processes, a preliminary initiative to study Brazilian dyads had also been made by Pessôa and Seidl-deMoura (2008). This preliminary study aimed to identify the pragmatic aspects of maternal input, focusing on the affective and cognitive contents of mothers' speech. Two groups were compared: dyads with one month-old babies and other with five months-old babies. They were observed in natural interactions at home, registered in videotapes. Results indicated that mothers' speech had predominantly affective content in both groups, confirming results found in research carried out in other cultures (Bornstein, Tal, \& Rahn, 1992). This study consisted in a restricted cross-sectional investigation. No Brazilian longitudinal studies of maternal speech transformations in specific communicative frames have been identified in the literature.

Trying to identify the individual development trajectories of socialization, Camaioni et al. (2003) had described these trajectories of four mother-child dyads. They have analyzed the transitions of communicative frames, observing these dyads (mothers of two boys and two girls) longitudinally, from 10 to 24 months of the children's life. Each dyad was filmed in a laboratory with specific toys, bi-weekly, for a period of ten minutes. Similarities and differences between the four dyads have been observed. Among them, it was verified the conventional frame/scenes and the representative gestures served as "bridge" between the attentional frame/scene and the symbolic frame/scene. Observations were organized in three age groups. It was observed that from the $44^{\text {th }}$ to the $66^{\text {th }}$ week all dyads had a predominance of attentional frames; from the $67^{\text {th }}$ to the $85^{\text {th }}$ week, attentional frames/scene also prevailed in dyads 1,2 and 4 , but conventional frames/scene increased substantially in terms of occurrence in time. In contrast, it was identified a predominance of conventional frame/scene for dyad 3. From the $86^{\text {th }}$ week on symbolic frames predominated. 
In Brazil, based on the study above, Pessôa and Seid-de-Moura (2011) also had described these trajectories of development in a longitudinal study to analyzed characteristics of maternal speech directed to children, identifying transitions of mother-child communication frames. Four children and their mothers were observed biweekly from 13 to 24 months of age. The instances of maternal speech were classified in affirmative, negative, imperative and interrogative sentences and the mother-child communication frames were classified in attentional, conventional or symbolic. The transitions from attentional to symbolic mother-child communication frames were also identified. A similar trajectory in the percentage of time of the frames throughout the development of the child was observed in all four dyads. Results indicate some common tendencies, which seem to be regulated by the child's development. In general, a developmental sequence was observed for the different frames in terms of their predominance: first attentional, then conventional and latter symbolic, in accordance to the literature (Camaioni et al., 2003). The percentage of time in attentional and symbolic frames showed a linear relation with the increase of baby's age in all dyads.

Mothers' communicative acts are an important part of this scenario. Mothers seem sensitive to the development of their children. They increase CDS linearly in relation to the child's age, use more declarative affirmative sentences and increase linearly the reference to aspects of the surrounding context of the dyad, decreasing the reference to the child him/herself. It seems that child's language development is the most important variable to explain the variations in the type and content of maternal sentences. The great diversity in the number of mothers' emissions indicates an adjustment to the specific characteristics of their children in this initial linguistic phase (Pessôa, 2008).

All these studies suggest that mothers adjust their behavior to the linguistic development of their children, in order to call their attention to themselves, to what they are speaking and, mainly, to the surrounding context. The significant increase in maternal speech related to the context, throughout the development of the child, seems to indicate that mothers gradually understand what is happening as their children develop across the second year of age.

Mothers make use of language, through different types of sentences (affirmative, imperative and negative) to communicate to their children and to transmit norms, rules, expected behaviors, and information about the world. The use of imperatives, throughout the period studied, can indicate an attempt of mothers to organize children's behaviors, besides being a verbal form to interact to children. The occurrence of interrogative emissions is another indicative of the establishment of linguistic interaction that, along with pauses, create turn taking, which is basic in a process of verbal interaction.

Intuitively, mothers use linguistic resources, through affirmative, negative, imperative and interrogative sentences, to call or to keep the attention of the child directed to them and the situations that involve them. This specific characteristic of maternal sentences has an evolutionary origin (Tomasello, 2003). After all, the necessary condition to establish a verbal communication between the mother-infant dyad is that the child has the attention directed to the mother and that they share attention to the context around them. 
Data from the dyads observed in Brazilian studies (Pessôa \& Seidl-de-Moura, 2008; Pessôa \& Seidl-de-Moura, 2011) indicated that mothers of these studies talk to their children about the situations in which they are involved. They speak about the babies themselves, about the activities carried out jointly by them, and, mainly, about the things and people in the context around them. However, it was possible to observe that almost all maternal emissions are preceded or followed by the children's signals. This means that mothers are not simply communicating information to their children, but are trying to engage them in dialogues. During the initial development, parents are the social agents who better understand children's intentions, attributing meanings to their behaviors, and providing the adequate support they need. Hence, they play an important role as mediators in the construction of children's social and cultural world.

As Keller (2007) points out, one of the components in the parental system of care is the narrative envelope. Babies are wrapped in this narrative envelope and their development processes are immersed on it, including language acquisition. A recent Brazilian research (Pessôa, Seidl-deMoura, Ramos and Mendes, submitted) aimed to verify parenting values among different young children's caretakers, comparing the speech of differents caretakers (mothers, grandmother, nanny and day-care center teacher) taking into account Keller's (2007) parental care systems (primary care, body contact, body stimulation, object stimulation and face to face interaction). The categories affection and physical contact were some of the most mentioned by all participants. These categories were associated with body contact and primary care systems. In all of the system it was found the category of affection. These analyses have served to illustrate that the affective aspects of speech, those which communicate affection and intimacy, were present in caretaker's speeches since early stages of development. Also we must consider the specific cultural characteristics that may be related to these findings. Different cultures may have different forms of communication and affective manifestations, and can vary in relation to the assigned value to each of these aspects in different moments of ontogeny.

As mentioned, according to a socio-cultural perspective to study human behavior, one cannot separate it from people's social and cultural contexts. Each culture has specific characteristics that need to be taken into account. Based on these assumptions, we believe in the importance of studying universal processes, such as initial language development and characteristics of maternal input in different cultures, to understand what may vary in the trajectories of these processes and what does not. The goal in these studies was to work with data from Brazilian dyads, Portuguese speakers and the trajectories observed, with the advantage of being a longitudinal investigation. Although not frequently used, longitudinal studies are particularly important. So, these studies bring a contribution to the literature on maternal speech and initial language development with data from a Brazilian context.

Most of the studies presented in this chapter indicated that a large proportion of maternal speech occurs in response to signals from the child, and this seems to mean that mothers are not simply passing on information to their children, but are trying to engage them in conversations and giving affection. Adopting some specific hypotheses on the philogenetic and ontogenetic origins of the language, Tomasello (2003) suggests that the symbolic dimension of the language has its origin in the singular character of human biological adaptation for 
cultural aspects. As we can think, the ability of caregivers to adjust his speech addressed to the baby seems not only to reflect a trait related to social and cultural beliefs, and related to the knowledge they have about development in early stages, but it also seems to be part of the baggage of predispositions of our species.

\section{Some final considerations}

Our purpose in this chapter was to present and discuss theoretical and empirical evidence about the role of affection and emotion in parental care and its impacts in the early development. It was particularly taken into account, in addition to emotional development, a cognitive process, language acquisition. It should also be highlighted researchers' concern to underline the diversity of ecological and social-cultural contexts in which the studies had been developed, as well as attention to the phylogeny.

According to an evolutionary view, complex behaviors such as language and emotional expressions rarely appear in an abrupt way and seldom present drastic modifications in a short period of time. It is plausible to think that they had their origins in other previous behaviors and simple biological mechanisms during a slow evolutionary history. Humans are social beings immersed in the socio-cultural context in which they live and are constituted by interacting with others. Therefore, some hypotheses for the origins of language, such as discussed by D. Falk (2004), even include the demands of child care in the environment of evolutionary adaptation and the motherese also can be seen as the result of selective pressures of our ancestral past. According to this view, the selection of vocal language occurred after the first mothers in hominids began to engage in emotional vocalizations directed to their babies. Emotional expressions and affective interactions can also be seen as adaptive processes that were selected by evolutionary mechanisms.

Affection and emotional expressions of the mother are important mediators for both emotional development and for language acquisition. As shown by the evidence presented in this chapter, particularly those regarding the Brazilian mothers residing in Rio de Janeiro, the dynamics of the mother-infant interactions from the beginning of life is characterized by adjustments in maternal behaviors, both emotional expression and speech, according to the possibilities and needs of the baby. Mothers intuitively adjust their speech directed to the baby and his emotional expression in order to promote communication between them and the baby's learning of such mechanisms of expression and interpersonal exchanges. In the Brazilian context, the parental ethnotheories are targeted for the presence of affective components on maternal speech and emotional contingency on mother-infant interaction, from the beginning of life.

It is important to bear in mind that the baby since birth has contact with emotional expressions and affective manifestations of his mother during the interactions established between both, through emotional manifestations of the mother in the body and face and through her speech direct to the baby. This is a cultural trait and how babies learn about others and about themselves. This way, it's reasonable to think that emotion, affection and maternal speech in 
parental care came together to constitute developmental trajectories for healthy children. We hope that this chapter may act as a motivating factor for future studies focusing on affection and emotion in parental care and its relevance to child development.

\section{Author details}

Deise Maria Leal Fernandes Mendes and Luciana Fontes Pessôa

Universidade do Estado do Rio de Janeiro, Pontifícia Universidade Católica do Rio de Janeiro, Brazil

\section{References}

[1] Bornstein, M. H., Cote, L. R., Maital, S., Painter, K., Park, S., Pascual, L., Pêcheux, M., Ruel, J., Venuti, P., \& Vyt, A. (2004). Cross-Linguistic Analysis of Vocabulary in Young Children: Spanish, Dutch, French, Hebrew, Italian, Korean, and American English. Child Development, 75 (4), 1115-1139.

[2] Bornstein, M. H., Tal, J., \& Rahn, C. (1992). Functional analysis of the contents of maternal speech to infants of 5 and 13 months in four cultures: Argentina, France, Japan, and United States. Development Psychology, 28 (4), 593-603.

[3] Bowlby, J. (1969), Attachment and loss, Vol. 1: Attachment. New York: Basic Books.

[4] Bronfenbrenner, U. (1996). A ecologia do desenvolvimento humano: experimentos naturais e planejados. Porto Alegre: Artes Médicas.

[5] Camaioni, L., Aureli, T., Bellagamba, F., \& Fogel, A. (2003). A Longitudinal Examination of the Transition of Symbolic Communication in the Second Year of Life. Infant and Child Development, 12 (2), 1-26.

[6] Camaioni, L., \& Longobardi, E. (2001). Noun versus verb emphasis in Italian motherto-child speech. Journal of Child Language, 28, 773-785.

[7] Chapman, R. S. (2000). Children's Language Learning: An Interactionist Perspective. Journal of Child Psychology and Psychiatry, 41, 33-54.

[8] Cole, P. M., \& Tamang, B. L. (1998). Nepali children's ideas about emotional displays in hypothetical challenges. Developmental Psychology, 34, 640-646.

[9] Cole, P. M., Tamang, B. L., \& Shrestha, S. (2006). Cultural Variations in the Socialization of Young Children's Anger and Shame. Child Development, 77(5), 1237-1251. 
[10] Cunha, I. da (2002). A base biomolecular das emoções. Em J. Wilheim (org.), Relação mãe-feto: visão atual das neurociências/Humanização do nascimento (pp. 83-108). São Paulo: Casa do Psicólogo.

[11] Denham, S. A., Workman, E., Cole, P. M., Weissbrod, C., Kendziora, K. T., \& ZahnWaxler, C. (2000). Prediction of externalizing behavior problems from early to middle childhood: The role of parental socialization and emotion expression. Development and Psychopathology., 12, 23-45.

[12] Denham, S., \& Kochanoff, A. T. (2002). Parental Contributions to Preschoolers' Understanding of Emotion. Em R. A. Farbes (Eds.), Emotions and the Family (pp. 311-343). NY: The Haworth Press, Inc.

[13] Dix, T. (1991). The Affective Organization of Parenting: Adaptive and Maladaptative Processes. Psychological Bulletin, 110(1), 3-25.

[14] Eisenberg, N. Spinrad, A., \& Cumberland, T. L. (1998). Parental Socialization of Emotion. Psychologycal Inquiry 9(4), 241-273.

[15] Falk, D. (2004). Prelinguistic evolution in early hominins: Whence motherese? Behavioral and Brain Sciences (27), 491-541.

[16] Harkness, S., \& Super C. M. (1994). The developmental niche: a theoretical framework for analysing the household production of health. Social Science and Medicine, 38 (2), 217-226.

[17] Harkness, S., Super, C. M., Bermudez, M. R., Moscardino, U., Rha, J. H., Mavridis, C. J. et al. (2010). Parental ethnotheories of children's learning. In D. F. Lancy, J. Brock, \& L.S. Gaskins (Eds.), The anthropology of learning in childhood (pp.65-81). Walnutcreek, CA: Alta Mira Press.

[18] Harlow, H. F. (1958). The Nature of Love. American Psychologist, 13, 673-685.

[19] Hoff, E., \& Naigles, L. (2002). How children use input to acquire a lexicon. Child Development, 73 (2), 418-433.

[20] Holodynski, M., \& Friedlmeier, W. (2010). Development of Emotions and Emotion Regulation. NY: Springer.

[21] Keller, H. (2007). Cultures of infancy. Mahwah, NJ: Lawrence Erlbaum.

[22] Keller, H., Borke, J., Yovsi, R., Lohaus, A., \& Jensen, H. (2005). Cultural orientations and historical changes as predictors of parenting behaviour. International Journal of Behavioral Development, 29 (3), 229-237.

[23] Keller, H., Borke, J., Lamm, B., Lohaus, A., \& Yovsi, R. D. (2011). Developing patterns of parenting in two cultural communities. International Journal of Behavioral Development, 35(3), 233-245. 
[24] Lewis, M. (2010). The Emergence of Human Emotions. In M. Lewis, J. M. HavilandJones \& L. F. Barrett (Eds.) Handbook of Emotions, 3nd Edition (pp. 304-319). NY: The Guilford Publications.

[25] Lidz, J., Gleitman, H., \& Gleitman, L. (2003). Understanding how input matters: verb learning and the footprint of universal grammar. Cognition, 73, 135-176.

[26] Lunkenheimer, E. S., Shields, A. M., \& Cortina, K. S. (2007). Parental Emotion Coaching and Dismissing in Family Interaction. Social Development, 16(2), 232-248. doi: 10.1111/j.1467-9507.2007.00382.x

[27] McElwain, N. L., Halberstadt, A. G., \& Volling, B. L. (2007). Mother- and Father-Reported Reac tions to Children's Negative Emotions: Relations to Young Children's Emotional Understanding and Friendship Quality. Child Development, 78(5), 1407-1425.

[28] Mendes, D. M. L. F., Seidl-de-Moura, M. L., \& Siqueira, J. O. (2009). The ontogenesis of smiling and its association with mothers' affective behaviors: a longitudinal data. Infant Behavior and Development, 445-453. Doi:10.1016/j.infbeh.2009.07.004.

[29] Mendes, D. M. L. F., \& Cavalcante, L. I. C. (submitted). Modelos de self e expressão emocional em bebês: concepções de mães e outras cuidadoras. Psico.

[30] Mendes, D. M. L. F., \& Seidl-de-Moura (submitted). Expression of smiling in infants up to six months: different types and contingency to maternal behaviors. The Spanish Journal of Psychology.

[31] Messinger, D., Dondi, M., Nelson-Goens, G. C., Beghi, A., Fogel, A., \& Simion, F. (2002). How sleeping neonates smile. Developmental Science, 5, 48-54. Obtido em http://www.blackwell-synergy.com/ em agosto de 2005.

[32] Mirabile, S. P., Scaramella, L. V., Sohr-Preston, S. L., \& Robison, S. D. (2008). Mothers' Socialization of Emotion Regulation: The Moderating Role of Children's Negative Emotional Reactivity. Child Youth Care Forum, 38, 19-37. DOI: 10.1007/ s10566-008-9063-5

[33] Nicholson, D., \& Artz, S. (2006). Caregiving strategies for reducing aggression and violence in at-risk mothers and their babies. Child $\mathcal{E}$ Youth Care Forum, 35, 411-426. doi:10.1007/s10566-006-9025-8.

[34] Pessôa, L. F. (2008). Maternal speech in communicatives frames and the development of language: a longitudinal study. Unpublished PHD Dissertation. Rio of Janeiro, UERJ.

[35] Pessôa, L. F., \& Seidl-de-Moura, M. L. (2008) A análise da fala materna dirigida a bebês em duas etapas do desenvolvimento. Psicologia em Pesquisa, 2 (2), 74-86. 
[36] Pessôa, L. F., \& Seidl-de-Moura, M. L. (2011). Fala materna dirigida à criança em cenários comunicativos específicos: Um estudo longitudinal. Psicologia: Teoria e Pesquisa, 27(4), 439-447.

[37] Pessôa, L. F., Seidl-de-Moura, M. L., Ramos, D, \& Mendes, D. M. L. F. (submitted). Valorização dos sistemas de cuidados e características do discurso de diferentes cuidadores do Rio de Janeiro forjando trajetórias de desenvolvimento. Paidéia

[38] Piontelli, A. (1992). From Fetus to Child. NY: Brunner-Routledge.

[39] Rao, N., McHaleb, J. P., \& Pearson, E. (2003). Links Between Socialization Goals and Child-Rearing Practices in Chinese and Indian Mothers. Infant and Child Development, 12, 475-492. DOI: 10.1002/icd.341

[40] Reddy, V., \& Trevarthen C. (2004). What we learn about babies from engaging with their emotions. Zero to Three, 24, no. 3: 9-15.

[41] Seidl-de-Moura, M. L., \& Mendes, D. M. L. F. (2012). Human Development: The Role of Biology and Culture. In M. L. Seidl-de-Moura (Ed.), Human Development: Different Perspectives (pp. 3-18). Croatia: Intech.

[42] Tomasello, M. (2003). Constructing a language: A usage-based theory of language acquisition. Harvard: University Press.

[43] Vila, I. (1995). Aquisição de linguagem. Em: Coll, C.; Palácios, J. \& Marchesi, A. (Org.), Desenvolvimento psicológico e educação. Porto Alegre: Artes Médicas.

[44] Weizman, Z. O., \& Snow, C. E. (2001). Lexical output as related to children's vocabulary acquisition: Effects of sophisticated exposure and support for meaning. Developmental Psychology, 37 (2), 265-279. 
